\title{
Hitchcock's (2001) treatment of singular and general causation
}

\author{
Christian Jakob
}

Received: 22 April 2006/ Accepted: 8 August 2006/

Published online: 27 October 2006

(C) Springer Science+Business Media B.V. 2006

\begin{abstract}
Hitchcock (2001a) argues that the distinction between singular and general causation conflates the two distinctions 'actual causation vs. causal tendencies' and 'wide vs. narrow causation'. Based on a recent regularity account of causation I will show that Hitchcock's introduction of the two distinctions is an unnecessary multiplication of causal concepts.
\end{abstract}

Keywords Causal claims - General causation - Philosophy of science · Regularity theory $\cdot$ Singular causation

\section{Introduction}

Consider the two following claims:

(S) David's smoking caused him to develop lung cancer.

(G) Smoking causes lung cancer.

Traditionally (S) is understood as a singular causal claim, whereas $(\mathrm{G})$ is interpreted as a general causal claim. The feature ostensibly discriminating between the two types of statements is the absence or presence of a reference to an entity located in time and space: (S) refers to David's smoking, whereas claim (G) lacks a comparable reference.

The singular-general distinction seems to have a firm place in our everyday use of causal language as well as in science and philosophy. Philosophers dealing with the topic of causation usually specify which of the presumably two types of causation they are talking about. In science it is well known that the methodological requirements differ as regards the justification of claims like $(\mathrm{G})$ and $(\mathrm{S})$. Last but

C. Jakob (ه)

Institute of Philosophy and History of Science, University of Berne, 3012 Berne, Switzerland e-mail: jakobchristian@students.unibe.ch 
not least, our everyday causal talk includes claims of type (S) as well as claims of type $(\mathrm{G})$ and we seem to do quite well in (intuitively) differentiating between them.

All this does not necessarily mean, however, that the singular-general distinction is conceptually basic and important. After all, there is considerable disagreement among scholars of causation, for example, as to whether there are one, two, or even more types of causal relations corresponding to the two types of causal claims. ${ }^{1}$ Christopher Hitchcock, after having defended a one-type approach in Hitchcock (1995), meanwhile proposes a whole variety of causal relations. ${ }^{2}$ Two of those various types of causal relations have been introduced in his Causal Generalizations and Good Advice, where he diagnoses a severe conceptual confusion concerning the singular-general distinction: ${ }^{3}$

I now believe that the distinction between singular and general causation conflates two separate distinctions, and that this conflation has greatly impeded progress in the understanding of causal generalizations.

In the present paper I will first address the two distinctions mentioned by Hitchcock-'actual causation vs. causal tendencies' and 'wide vs. narrow causation'-and show how he thinks the singular-general distinction conflates them. ${ }^{4}$ Then, I will give a short summary of an up-to-date regularity theory of causation on the basis of which I will argue that Hitchcock's introduction of the two distinctions is an unnecessary multiplication of causal concepts.

\section{Hitchcock's distinctions}

Actual causation vs. causal tendencies

According to Hitchcock, 'to cause' is a success verb when used in claims about actual causation. What does it mean for a verb to function as a success verb? Consider the verbs 'to know' and 'to believe'. The former is a success verb, whereas the latter is not, because 'Jago knows that $P$ ' entails the truth of $P$, whereas 'Jago believes that $P$ ' does not. In claim (S), mentioned at the beginning of this paper, 'caused' is a success verb. That is, (S) entails that David indeed smoked (or still does) and that he actually developed lung cancer. Hence, $(\mathrm{S})$ is a statement about actual causation.

Hitchcock's first example of a claim about a causal tendency is: ${ }^{5}$

(T) David is the sort of person for whom smoking tends to cause lung cancer.

In contrast to statements about actual causation claims about causal tendencies do not imply anything about the actual occurrence of the named events: $(\mathrm{T})$ may still be true even if David never did nor will smoke and never did nor will develop lung cancer. The verb 'to cause' does not function as a success verb in causal tendency claims.

\footnotetext{
${ }^{1}$ See, for example, Sober (1985) and Eells (1991) for arguments in favor of two types of causal relations and Carroll (1991) for a one-type approach.

${ }^{2}$ See especially Hitchcock (2003).

${ }^{3}$ Hitchcock (2001a), p. 219.

4 The actual-tendency distinction is originally due to Good (1961) and (1962).

${ }^{5}$ Hitchcock (2001a), p. 220.

管 Springer
} 
Wide vs. narrow causation

The claims (S) and (T), although belonging to separate categories with respect to the actual-tendency distinction, nevertheless share a common feature: They are both narrow causal claims in that they do not explicitly refer to any individual other than David. (S) is a statement about narrow actual causation, whereas (T) is a claim about a narrow causal tendency. Following Hitchcock, narrow causal claims "describe a causal relationship that holds within an individual or a homogenous population". 6

Since, as Hitchcock asserts, the distinctions between actual causation vs. causal tendencies and narrow vs. wide causation cross-classify, there are still two types of causal statements which we have not considered yet. These are (i) claims about wide actual causation and (ii) claims about wide causal tendencies.

Hitchcock's example of claims of wide actual causation is: ${ }^{7}$

(W) Every year, there are thousands of new cases of lung cancer that are caused by smoking.

As is true of claim (S), statement (W) implies that the named events took place: There are, in fact, thousands of cases of lung cancer which are actually caused by smoking. Unlike (S), however, (W) does not apply to a single individual or a narrowly circumscribed type of individual only-it is far more general. Hence, for Hitchcock (W) is a claim about wide actual causation.

The remaining type of causal claims relating to the two distinctions is that of wide causal tendencies. Hitchcock's example has been already introduced at the beginning of this paper:

(G) Smoking causes lung cancer.

As statement $(\mathrm{T})$, claim $(\mathrm{G})$ does not imply any actual occurrences of smoking or lung cancer, hence it is a claim about causal tendencies. In contrast to $(\mathrm{T})$, however, there is no reference to a particular individual nor a homogenous class of individuals, so $(\mathrm{G})$ is a claim about a wide causal tendency.

The conflation

Back in 1995, Hitchcock stipulated the following thesis: ${ }^{8}$

Singular causal claims imply the occurrence of events of a certain sort, while general causal claims do not.

Today, however, his two new distinctions together with his notion of the singulargeneral distinction overthrow the cited thesis.

According to Hitchcock (personal communication) a causal relation is singular, if it involves particular individuals at particular places and times. By contrast, a causal relation is general, if it is independent of particular individuals at particular places and times. According to this characterization both (S) and (T) seem to be cases of statements about singular causation: Both (S) and (T) refer to the individual David, and-since David is a particular-particular (frames of) places and times are

\footnotetext{
${ }^{6}$ Hitchcock (2001a), p. 220.

7 Hitchcock (2001a), p. 220.

8 Hitchcock (1995), p. 283.
} 
intimately tied to the meaning of every claim involving David. Contrary to the 1995thesis, however, only claim (S) implies the occurrence of the named events. Hence, the singular-general distinction conflates the concepts of actual causation and causal tendencies.

Much the same seems to be true of the wide-narrow distinction: Intuitions similar to the ones suggesting that singular causation is always actual, may also suggest that singular causal statements are narrow in scope, whereas general causal statements are wide. According to Hitchcock's understanding of the singular-general distinction, however, the wide causation claim (W) is singular since it refers to causal relations holding between actual cases of smoking and lung cancer (even though they are not explicitly named). Hence, the singular-general distinction conflates the wide-narrow distinction as well. It does not seem possible to strictly differentiate between the four types of causal statements on the basis of the singular-general distinction as such.

\section{A regularity theory of causation}

The merits and limits of a distinction like the singular-general distinction are dependent on the clarity of the respective concepts, of course. In this section, Hitchcock's characterization of singular and general causation will be refined by introducing a recent regularity account. ${ }^{9}$

\section{Preliminaries}

The regularity theory MT essentially consists of two parts, which are (i) conceptual analysis and (ii) analysis of causal inferences. ${ }^{10}$ The principal aim of the conceptual analysis is to define the notions of causal relevance and singular causation. The analysis of causal inferences, in contrast, focuses on the kinds of assumptions one has to accept when justifying causal claims based on empirical data. In this paper, however, only a simplified sketch of the conceptual analysis will be provided. ${ }^{11}$

\section{Causal principles}

The definition of causal relevance within the framework of MT satisfies four causal principles. Those principles are thought to constitute a minimal core of criteria which any theory of deterministic causation should satisfy.

Principle of causal determinism: The same cause is always accompanied by the same effect.

If there are irreducibly probabilistic relations the MT-analysis does not apply to them.

\footnotetext{
${ }^{9}$ Regularity theories of causation face a standard set of objections such as the purported inability to identify epiphenomena and spurious correlations, and to account for the asymmetry of causal relations. Solutions to the mentioned objections (including the 'Manchester factory hooters' and more) are proposed in Graßhoff and May (2001).

10 The name of the theory, MT, derives from the first letters of the essential theoretical construct: Minimal Theory. At first sight, MT bears a close resemblance to Mackie's (1974) INUS-conditions, but a second glance will show that some important lessons have been learned.

${ }^{11}$ For the analysis of causal inferences as well as the details and formal representation of the conceptual analysis see Graßhoff and May (2001), Baumgartner and Graßhoff (2004), and Baumgartner (2005). 
The essence of the second principle is that effects do not occur spontaneously:

Principle of causality: If no cause is present, no effect occurs.

Note, that this principle does not state that every event has a cause. Rather, it claims that if an event type is considered to be a type of effect of some type of cause, then whenever an instance of the type of effect occurs there is an occurrence of at least one of its causes.

Whenever an effect occurs there is a host of event types instantiated, but not all of those events are indispensable for the effect to occur-some are mere coincidences. The third principle addresses our intuition that causal dependencies among events are more than mere coincidences:

Principle of causal relevance: Every type of cause is indispensable for the occurrence of an effect in at least one situation.

Inferences to causes of an effect are typically based on incomplete knowledge about the factors instantiated in a causally analyzed process. If additional features of the situations are taken into account we expect a cause to maintain its relevance. This expectation is captured by the fourth principle:

Principle of persistent relevance: An event type maintains its causal relevance when additional event types are taken into account.

\section{Causal relevance and singular causation}

Drawing on the four principles introduced in the previous section we may now define the concepts ultimately needed in order to analyze the notions of causal relevance and singular causation.

Minimally sufficient conditions $(\mathrm{MsC})$ : According to the Principle of Causal Determinism, a cause is sufficient for its effect: $C \rightarrow E .^{12}$ A material conditional is insensitive to considerations of relevance, however: From $C \rightarrow E$ one can, for example, derive $C \& D \rightarrow E$, whatever $D$ stands for. This violates the Principle of Causal Relevance. A minimality constraint imposed on sufficient conditions solves this problem:

MsC: A conjunctive sufficient condition $\Phi$ of an event type $\Theta$ is a minimally sufficient conjunction of $\Theta$, if and only if no proper part of $\Phi$ is sufficient for $\Theta$.

$\Phi$ and $\Theta$ are used as variables standing for simple or complex event types. ${ }^{13}$ 'Proper part of $\Phi^{\prime}$ means a conjunction $\Phi^{\prime}$ containing fewer conjuncts than $\Phi$ where every conjunct in $\Phi^{\prime}$ is a conjunct in $\Phi .{ }^{14}$ In Fig. $1 C X_{1}$ is a conjunctive minimally sufficient condition of $E$. $C$ refers to a single event type, whereas $X_{1}$ consists of a conjunction of simple event types. ' $C X_{1}$ ' is to be read as $C \& X_{1}$. If $C$ is interpreted in terms of 'smoking' then $X_{1}$ stands for a conjunction of event types including physiological properties of the smoker, properties of the tobacco, and so on. The arrows mean 'is

\footnotetext{
12 An adequate formal representation of causal claims requires first-order logic. For purposes of simplicity, however, quantifiers, variables, and non-causal relational properties of event types and events are omitted. As a consequence, material conditionals and bi-conditionals are to be read such that the event types in the antecedence are always coincidentally instantiated by events different from the event instantiating the consequence.

${ }^{13}$ Complex event types are conjunctions of simple event types.

${ }^{14}$ Note that event types redundant relative to one minimally sufficient condition may nonetheless be part of other minimally sufficient conditions for the same effect.
} 


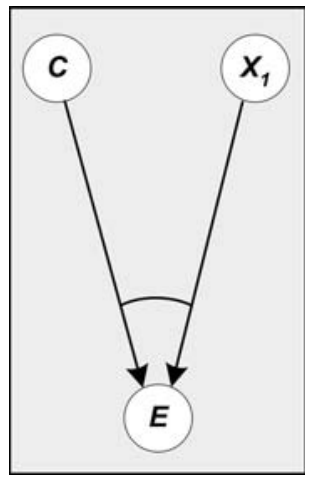

Fig. 1 The minimally sufficient condition $C X_{1}$ of $E$

causally relevant for' (the explication of which follows) and the arch between the arrows symbolizes the conjunction.

Minimally necessary conditions $(\mathrm{MnC})$ : The four principles allow alternative types of causes for a type of effect. Besides a minimally sufficient condition of lung cancer $S X_{1}$, which includes smoking $S$, there is, for example, also a minimally sufficient condition $A X_{2}$ not containing smoking, but inhaling asbestos $A$ and various further (known or unknown) alternative causes $Y$. Alternative causes of a type of effect are represented by a disjunction of (minimally) sufficient conditions: $C X_{1} \vee A X_{2} \vee Y \rightarrow E$.

According to the Principle of Causality, for every occurrence of a type of effect $E$ there is at least one of its causes present. Hence, the disjunction of all alternative causes of an effect is a necessary condition of the effect. This relation is not modeled by a material conditional, of course, but we can represent it with a bi-conditional: $C X_{1} \vee A X_{2} \vee Y \leftrightarrow E$.

If $A \vee B$ is necessary for $C$, so is $A \vee B \vee D$, whatever $D$ stands for. Hence, similarly to sufficient conditions, necessary conditions of an effect have to be subjected to a minimality criterion in order to satisfy the Principle of Causal Relevance:

MnC: A disjunctive necessary condition $\Psi$ of $\Theta$ is a minimally necessary con-

dition of $\Theta$, if and only if no proper part of $\Psi$ is necessary for $\Theta$.

'Proper part of $\Psi^{\prime}$ means a disjunction $\Psi^{\prime}$ containing fewer disjuncts than $\Psi$, where every disjunct in $\Psi^{\prime}$ is a disjunct in $\Psi$. In Fig. 2 the disjunction $C X_{1} \vee D X_{2} \vee Y$ is a minimally necessary condition of $E$. $C X_{1}$ and $D X_{2}$ are simple minimally sufficient conditions of $E$, and $Y$ is a complex minimally sufficient condition of $E$ consisting of a disjunction of simple minimally sufficient conditions. The absence of an arch between arrows symbolizes disjunction. A minimally necessary disjunction of minimally sufficient conditions of $E$ is called a minimal theory of $E$.

Causal relevance: A necessary condition of the causal relevance of an event type $C$ for an event type $E$ is the membership of $C$ in a minimal theory of $E$. $C$ 's being part of a minimal theory of $E$ is not sufficient for the causal relevance of $C$ for $E$, however. Minimal theories always are constructed by considering a restricted set of event types, i.e., the frame of event types of a minimal theory. Given certain causal structures such as epiphenomena, a later extension of the set of event types originally considered may affect the composition of an original minimal theory. In case a certain event type $D$ ceases to be part of a minimal theory of $E$ after the frame of 


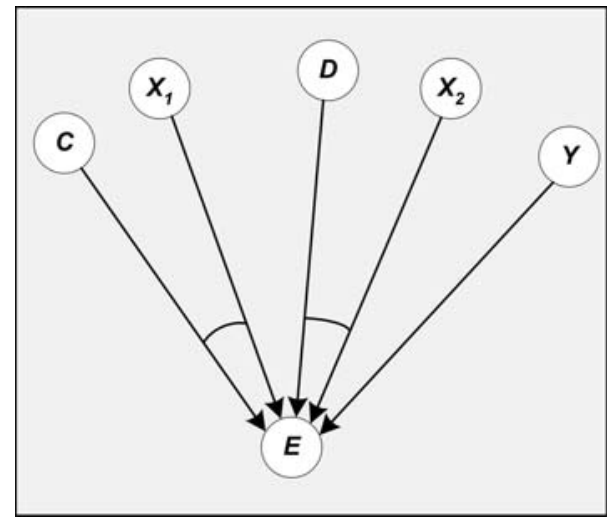

Fig. 2 A minimal theory of $E$, consisting of the minimally necessary condition $C X_{1} \vee D X_{2} \vee Y$

event types has been extended, $D$ is not causally relevant to begin with. This implementation of the Principle of Persistent Relevance enters the definition of causal relevance:

CR: An event type $C$ is causally relevant for an event type $E$ if and only if (i) $C$ is part of a minimal theory of $E$, and (ii) $C$ stays part of this minimal theory across any extensions of its frame of event types. ${ }^{15}$

Singular causation: In contrast to general causation, singular causation is a relation between entities located in time and space:

SC: Two events $c$ and $e$ stand in a singular causal relation if and only if $c$ instantiates a positive event type $C$ which, according to $\mathbf{C R}$, is causally relevant for an event type $E$, such that $e$ instantiates $E$, and $c$ is coincident with other events that instantiate a minimally sufficient condition $C X$ of $E$, in which $C$ is contained.

In the framework of MT, prevention and 'causation' by omission are not considered to be cases of genuine singular causation. Rather, claims about prevention and 'causation' by omission express explanatory relations which are relations between facts. ${ }^{16}$

\section{Hitchcock's distinctions revisited}

Equipped with the basic concepts of MT let us revisit Hitchcock's distinctions. ${ }^{17}$

Actual causation vs. causal tendencies

As to the relation between the singular-general distinction and actual causation vs. causal tendencies Hitchcock's (2001) conclusion is that singular causation, in

\footnotetext{
${ }^{15}$ In fact, (CR) is the definition of direct causal relevance. Indirect causal relevance is also defined within the framework of MT, but the distinction of direct vs. indirect causal relevance is of no importance for the subject of this paper.

${ }^{16}$ For similar views see for example Davidson (1967), p. 702f and Dowe (2001).

${ }^{17}$ The following critique of Hitchcock's distinctions can also be stated on the basis of theories of causation other than MT.
} 
contrast to his 1995-thesis, is not always actual causation (see p. 3). The most relevant example is claim ( $\mathrm{T}$ ) which, according to Hitchcock's characterization, is a statement about singular causation, because it involves a particular individual (i.e., David), but nonetheless does not imply anything with respect to the actual occurrence of the named events.

For a claim to be about a singular causal relation in the sense of MT a statement has to refer to at least two events standing in a causal relation (see $\mathbf{S C}$ on p. 7). This is not the case for $(\mathrm{T})$ :

(i) Smoking $S$ is causally relevant for lung cancer $L$.

(ii) David instantiates a conjunction of event types $X$ together with which smoking is a minimally sufficient condition $S X$, which is part of a minimal theory of $L$.

Part (i) of the Minimal Theorist's explication of (T) explains why (T) does not imply anything about David's actual behavior regarding smoking and the developing of lung cancer: (i) is only a claim about the general causal relation between smoking and lung cancer and, according to $\mathbf{C R}$, claims about general causation do not imply anything about particular instances of event types (see p. 7). The Principle of Causal Relevance requires that there is at least one situation in which an instance of smoking is indispensable for lung cancer to occur, but it does not matter which particular situation this is. ${ }^{18}$ Part (ii), in turn, accounts for the fact that (T) includes a reference to a particular individual without stating a singular causal relation: (ii) claims that David instantiates a certain conjunction of event types $X$ but not that he instantiates $S X$ and $L$ as SC would require for (T) to express a singular causal relation (see p. 7$).{ }^{19}$

One might argue against the MT-analysis of the meaning of (T) by claiming that the conjunction of (i) and (ii) does not explicate statements like (T) but rather statements of the following type:

$\left(\mathrm{T}^{\prime}\right)$ David is the sort of person for whom smoking causes lung cancer.

Claim ( $\left.\mathrm{T}^{\prime}\right)$ differs from $(\mathrm{T})$ only in that the expression 'tends to cause' is replaced with 'causes'. The two statements should therefore be a paradigmatic case of the distinction between actual causation and causal tendency. If the difference between (T) and $\left(\mathrm{T}^{\prime}\right)$ could be explicated with reference to Hitchcock's actual-tendency distinction, however, $\left(\mathrm{T}^{\prime}\right)$ should imply the actual occurrence of David's smoking and developing of lung cancer. But it does not. Possibly, the occasional occurrence of the verb 'to tend' in causal claims simply stresses the fact that such claims are not statements about singular causation. Another possibility would be that (T), in contrast to $\left(\mathrm{T}^{\prime}\right)$, refers to something like indeterministic causation. This, however, would not have anything to do with the thesis about the conflation of the actual-tendency distinction with the singular-general distinction.

\footnotetext{
${ }^{18}$ Even Hitchcock himself assumes that on type-level, i.e., general causation, the verb 'to cause' always describes causal tendencies rather than cases of actual causation (see Hitchcock (2001b), p. 375 , footnote 9 ).

${ }^{19}$ The difference between Hitchcock's classification of (T) as a narrow causal tendency and its MTanalysis may be considered as merely terminological, concerning what 'singular causal statement' is to mean. The fact that, according to Hitchcock's account of singular vs. general causation, (T) is a claim about singular causation even though the only causal relation expressed is one between types of events, however, seems to be a terminological oddity worth criticizing.
}

Springer 
In contrast to $(\mathrm{T})$, claim $(\mathrm{S})$ is interpreted as a statement about singular causation within MT:

(i) Smoking $S$ is causally relevant for lung cancer $L$, and

(ii') David instantiates a minimally sufficient condition $S X_{i}$ containing smoking $S$, and $S X_{i}$ is part of a minimal theory of $L .^{20}$

There are two particular events, David's smoking and his developing of lung cancer, respectively, which are claimed to stand in a singular causal relation as explicated by SC. Events are spatiotemporally located entities. ${ }^{21}$ Hence, every claim about singular causation implies the actual occurrence of the named events because of the very notion of an event.

Though not as typical as claim (S), statement (W) is a claim about singular causation, too:

(i) Smoking $S$ is causally relevant for lung cancer $L$, and

(ii") Every year, thousands of people instantiate a minimally sufficient condition $S X_{i}$ containing smoking $S$, and $S X_{i}$ is part of a minimal theory of $L$.

There are, in fact, thousands of causally related cases (i.e., spatiotemporally located entities) of smoking and lung cancer every year. Any additional categorization of (W) in terms of the actual-tendency distinction is superfluous, since all claims about singular causation are claims about actual causation.

Claim (G), finally, is an example of a statement about general causation as prototypical as claim $(\mathrm{S})$ is an example of a statement about singular causation:

(i) Smoking $S$ is causally relevant for lung cancer $L$.

(G) states a causal relation between two types of events, i.e., smoking and lung cancer, respectively. According to the MT-analysis of causal relevance $\mathbf{C R}$, it contains absolutely no explicit or implicit reference to particular instances of the mentioned types of events, and as such does not imply anything about particular occurrences of events of the mentioned types. Again, the actual-tendency distinction does not further illuminate the meaning of the claim, since being a claim about general causation implies not being a claim about actual causation. ${ }^{22}$

Let us now turn to the other two types of causation which, according to Hitchcock (2001), are conflated with the singular-general distinction, too.

Wide vs. narrow causation

Both (S) and (W) fall into the category of claims about singular causal relations. Yet, according to Hitchcock they differ with respect to the distinction of narrow vs. wide

${ }^{20}$ David also instantiates $L$, of course, but this follows from David's instantiation of $S X_{i}$.

${ }^{21}$ This seems to be true according to any arbitrary theory of events.

${ }^{22}$ I'm fully aware that $\mathbf{C R}$ as the analysis of $(G)$ does not explicate the undoubted practical and conceptual connections between general causal claims like (G) and recommendations for interventions (i.e., 'Refrain from smoking if you do not want to develop lung cancer'). The MT-account of those connections will, of course, refer to CR, but such an analysis of (recommendations for) interventions is considered to be a task separate from the analysis of claims like $(G)$ in terms of CR. 
causation: (S) states a causal relation that holds within a particular individual, i.e., David, whereas (W) describes a causal relation that holds within a broader, more heterogenous population.

The claim about the general causal relation between smoking and lung cancer (i) which is part of the meaning of $(\mathrm{S})$ is restricted neither to a particular individual nor a homogenous population: It only states that there is at least one combination of causal conditions for lung cancer of which smoking is, according to CR, a nonredundant part. As can be seen when comparing the respective MT-analyses of (S) and $(\mathrm{W})$ in the previous section, the same proposition (i) is also part of the meaning of (W). Since the causal relevance claim concerning smoking and lung cancer (i) is exactly the same in the analysis of (S) and (W) it should be clear that the scope of the therein claimed (general) causal relation does not differ between (S) and (W). If we do state the causal relevance of a certain type of event for another, e.g. smoking is causally relevant for lung cancer, we neither make an implicit nor an explicit claim about the frequency with which particular instances of smoking actually cause instances of lung cancer in different populations. ${ }^{23}$

The difference between claim (S) and (W) arises only in the second parts of the respective analyses which in conjunction with the respective first parts account for (S) and (W) being statements about singular causation: In (ii') there is only one single individual, i.e., David, which is claimed to instantiate a minimally sufficient condition of lung cancer such that his smoking is to be regarded as a cause of his lung cancer. Claim (ii"), in contrast, refers to thousands of people instantiating some minimally sufficient condition of lung cancer such that their smoking is to be regarded as a cause of their lung cancer. Note, that it is possible that all of the thousands of people referred to in (W) as well as David instantiate exactly the same minimally sufficient condition $S X_{i}$ of lung cancer. This would mean that it is exactly the same causal regularity which is instantiated by David and the thousands of people, respectively. The essential qua necessary difference between (S) and (W) is the mere number of instantiations which is one in $(\mathrm{S})$ and thousands in (W). This difference has nothing to do with the nature of the causal relations expressed in (S) and $(\mathrm{W})$ as the distinction between wide and narrow causation wrongly suggests. The causal relations expressed in (S) and (W) are of one and the same kind: singular causation.

\section{Conclusion}

MT, in contrast to Hitchcock's account, does not postulate more than two (interrelated) types of causal relations, i.e., causal relevance and singular causation. This is not to say, however, that there are only two types of causal statements. Yet, whereas Hitchcock introduces four causal relations in order to cope with four types of causal claims, MT is able to differentiate between those causal statements on the basis of the traditional two types of causation alone. For the sake of Ockham's razor Hitchcock's four types of causal relations are to be dismissed in favor of the singular-general distinction as explicated by MT.

${ }^{23}$ It seems to me that the very basic idea of probabilistic analyses of causation, i.e., probabilityraising of effects by their causes, promotes exactly this confusion between the existence of a general causal relation and the frequency of its instantiation in certain populations. 
Acknowledgements For comments on earlier drafts I would like to thank Michael Baumgartner, Gerd Graßhoff, Christopher Hitchcock, audience members at the University of Berne (Switzerland) and the University of Konstanz (Germany), and anonymous referees.

\section{References}

Baumgartner, M. (2005). Complex causal structures: Extensions of a regularity theory of causation. Ph.D. thesis, University of Berne.

Baumgartner, M., \& Graßhoff, G. (2004). Kausalität und kausales Schliessen. Bern: Bern Studies in the History and Philosophy of Science.

Carroll, J. (1991). Property-level causation. Philosophical Studies, 63, 245-270.

Davidson, D. (1967). Causal relations. The Journal of Philosophy of Science, 64(21), 691-703.

Dowe, P. (2001). A counterfactual theory of prevention and 'Causation' by omission. Australasian Journal of Philosophy, 79(2), 246-226.

Eells, E. (1991). Probabilistic causality. Cambridge: Cambridge University Press.

Good, I. J. (1961). A causal calculus I. British Journal for the Philosophy of Science, 11, 305-318.

Good, I. J. (1962). A causal calculus II. British Journal for the Philosophy of Science, 12, 43-51.

Graßhoff, G., \& May, M. (2001). Causal regularities. In: Spohn W., Ledwig M., \& Esfeld M. (Eds.), Current issues in causation. Paderborn: Mentis.

Hitchcock, C. (1995). The mishap at Reichenbach fall: Singular vs. general causation. Philosophical Studies, 78, 257-291.

Hitchcock, C. (2001a). Causal generalizations and good advice. The Monist, 84, 222-246.

Hitchcock, C. (2001b). A tale of two effects. The Philosophical Review, 110(3), 361-396.

Hitchcock, C. (2003). Of humean bondage. British Journal for the Philosophy of Science, 54, 1-25.

Mackie, J. L. (1974). The cement of the universe. A study of causation. Oxford: Clarendon Press.

Sober, E. (1985). Two concepts of cause. In: Asquith P., \& Kitcher P. (Eds.), PSA 1984 (pp. 405-425) Vol. 2. East Lansing: Philosophy of Science Association. 